\title{
RADIO OBSERVATIONS OF THE LUNAR ATMOSPHERE
}

\author{
B. ELSMORE \\ Mullard Radio Astronomy Observatory, Cavendish Laboratory \\ Cambridge, England
}

Observations of a lunar occultation of a radio source may provide information concerning both the distribution of radio "brightness" across the source and its accurate position. For sources of which these results are already fairly well known, observations at long wavelengths may be used to derive the density of the lunar atmosphere [1]. During recent years two such occultations have been observed at Cambridge: one, the occultation of IC 443, the large-diameter radio source in the constellation of Gemini, from which the density of the lunar atmosphere was estimated to be less than $10^{-12}$ of that of the density of the terrestrial atmosphere [2] and [3]; and two, the occultation of the Crab nebula on 1956 January 24 [4].

Accurate observations were made at a wavelength of $3.7 \mathrm{~m}$ throughout the occultation of the Crab nebula, from which a curve was plotted of the observed radio-emission intensity. Since the moon's center passed near the center of the source, different points on the nebula were covered and uncovered in the same sequence. The occultation curves obtained during emersion and immersion were therefore complementary, and corresponding points on the curve were found to be separated by an interval of $59 \mathrm{~m} 6 \pm 0.26$. The calculated time of obscuration, $59 \mathrm{~m} 21$, is $0 \mathrm{~m}^{\mathrm{m}} \pm 0.26$ less than the observed time.

This difference between the observed and the calculated times is attributed to refraction in the sunlit ionized atmosphere of the moon and corresponds to angle of $13^{\prime \prime} .4 \pm 8.7$, if it is assumed that refraction occurred only at the sunlit limb. Although this represents a barely significant result, it will be assumed for the present to have a meaning in deriving a value for the electron density; the observations, in any case, provide a figure for the maximum permissible density.

There are difficulties in estimating the chemical composition, temperature, and, hence, the scale height in the lunar atmosphere, but if it is assumed that the temperature of the atmosphere is not very different from that of the sunlit surface $\left(380^{\circ} \mathrm{K}\right)$, the scale height of a "permanent" lunar atmosphere would be about $50 \mathrm{~km}$ and the electron density required to produce the observed angle of refraction is $10^{3} \mathrm{~cm}^{-3}$. A similar value is obtained if the atmosphere is not permanent but consists of gases that are continuously escaping and that are being replaced at the moon's surface. This escaping atmosphere might come from $(a)$ radioactive decay of part of the rocks comprising the moon's crust, $(b)$ vaporization produced by the impact of meteors 
striking the moon's surface, or $(c)$ release of primeval gases from the moon's interior.

Assuming that about one molecule in a thousand in the lunar atmosphere is ionized, the density of the atmosphere at the moon's surface is about $2 \times 10^{-13}$ of the density of the earth's atmosphere at normal temperature and pressure, and, taking 22 seconds of arc as the upper limit of the angle of refraction, it is unlikely to exceed $6 \times 10^{-13}$ atmospheres at N.T.P.

These observations, which are discussed in detail elsewhere [5], provide a measurement over a thousand times more sensitive than the upper limit derived by Dollfus [6] from optical measurements. When the accurate declinations of more radio sources in the zodiacal band are known, it may be possible to repeat the measurements, and probably when the Crab nebula is again occulted in the period 1963-64. The value of observations at long wavelengths must be emphasized, since refraction increases with the square of the wavelength.

\section{REFERENCES}

[1] Link, F. Bull. Astr. Insts. Csl. 7, 1, 1956.

[2] Elsmore, B., and Whitfield, G. R. Nature, 176, 457, 1955.

[2] Elsmore, B. Radio Astronomy (I.A.U. Symposium No. 4, 1955). Cambridge, England, 1957, Ch. 78.

[4] Costain, C. H., Elsmore, B., and Whitfield, G. R. M.N.R.A.S. 116, 380, 1956.

[5] Elsmore, B. Phil. Mag. Ser. 8, 2, 1040, 1957.

[6] Dollfus, A. C.R. 234, 2046, 1952.

\section{Discussion}

Zirin: Since the ionized hydrogen region of the sun must extend beyond one astronomical unit, the atmosphere of the moon ought to be entirely ionized; thus, one should consider the number of atoms as equal to the number of electrons. The densities arrived at by Elsmore should be reduced by $10^{2}$ or $10^{3}$.

Elsmore: Recombination at the lunar surface may cause only 0.1 per cent to be ionized, but if this is not so, then the lunar atmosphere must have a density as low as $10^{-10}$ of the terrestrial atmosphere.

Vitkevich: Udal'tsov and Vitkevich at the Crimean Station of the Physical Institute of the U.S.S.R. have received radio waves from the Crab nebula on a wavelength of 3.5 and $5.8 \mathrm{~m}$ during an eclipse of the moon. The refraction in the lunar atmosphere was observed to be not larger than 2 minutes of arc at these wavelengths.

Kaiser: Dr. Elsmore's lunar electron density is of the same order as some estimates of interplanetary electron density at the earth's distance from the sun. Has the author considered that the perturbation in this electron density may be caused by hydrodynamic flow of the ionized interplanetary gas around the moon? 
Elsmore: I have considered this and find that it is unlikely to be important. I must point out that the $10^{3}$ electrons per cubic $\mathrm{cm}$ measured is the excess over that in the surrounding medium.

Hoyle: Is this not for the maximum refractive effect, when there is no cancellation of the 13 seconds of arc by a contribution from the probable error, which is nearly as large?

Gold: The question of motion through the interplanetary gas is an extremely difficult one and would surely have been worthy of a separate paper.

Elsmore: I do not consider the problem to be one of hydrodynamics, but one of individual impacts of ionized gas molecules with a nonconducting surface. I sought the aid of Professor Mott, and believe the problem has been solved.

Dewhirst: Does the theoretical duration of occultation depend on the assumed place of the radio center of gravity of the Crab nebula?

Elsmore: No, because the occultation was almost a central one in this case.

Link: The calculation of the electron density in the lunar atmosphere depends on the structure of this atmosphere. One could investigate the influence of this more or less theoretical structure on the density calculation. It appears that, thanks to the form of the refraction integral, it is principally the electron density at the lowest point of the ray that makes itself felt first. The actual structure of the atmosphere can vary the final result only about ten to one, and thus cannot change our opinion on the almost perfect "vacuum" that reigns on the moon. 